\title{
Modeling pancreatic cancer with organoids
}

Lindsey A. Baker ${ }^{1,2}$, Hervé Tiriac ${ }^{1,2}$, Hans Clevers ${ }^{4}$, David A. Tuveson ${ }^{1,2,3 *}$

${ }^{1}$ Cold Spring Harbor Laboratory, Cold Spring Harbor, NY 11724, USA

${ }^{2}$ Lustgarten Pancreatic Cancer Research Laboratory, Cold Spring Harbor, NY 11724, USA

${ }^{3}$ Rubenstein Center for Pancreatic Cancer Research, Memorial Sloan Kettering Cancer Center, New York, NY 10065, USA

${ }^{4}$ Hubrecht Institute and University Medical Centre Utrecht, Utrecht, The Netherlands

*Corresponding author: Tuveson, D.A. (dtuveson@ cshl.edu) 


\section{ABSTRACT}

3 Pancreatic ductal adenocarcinoma (PDA) is a highly lethal malignancy for which new treatment

4 and diagnostic approaches are urgently needed. In order for such breakthroughs to be discovered,

5 researchers require systems that accurately model the development and biology of PDA. While

6 cell lines, genetically engineered murine models, and xenografts have all led to valuable clinical

7 insights, organotypic culture models have emerged as tractable systems to recapitulate the

8 complex three-dimensional organization of PDA. Recently, multiple methods for modeling PDA

9 using organoids have been reported. This review aims to summarize these organoid methods in

10 the context of other PDA models. While each model system has unique benefits and drawbacks,

11 ultimately, organoids hold special promise for the development of personalized medicine

12 approaches. 


\section{The Need for Accurate Model Systems of Pancreatic Cancer}

14 Pancreatic cancer is an extremely lethal malignancy, with a 5-year survival rate of less than $7 \%$

15 [1]. This malignancy is currently the fourth leading cause of cancer deaths in the United States

16 [1] and is projected to surpass colon and breast cancers by 2030 [2]. Pancreatic ductal

17 adenocarcinoma (PDA), the most common form of pancreatic cancer, is often diagnosed too late

18 for effective therapeutic intervention to succeed, and only $10-15 \%$ of patients diagnosed with

19 PDA are eligible for surgery, the only potentially curative option [3]. Thus, new approaches to

20 diagnose and treat PDA are urgently needed.

22 In order for clinical progress to be made, researchers need accurate and tractable model systems

23 for studying this malignancy. At first glance, it would seem that the best way to study PDA

24 would be to use human tumor tissue. However, the use of such tissue has limitations as a model.

25 Human tumors are often diagnosed late in tumor evolution, providing a snapshot of a late stage

26 of tumorigenesis, and offering little utility to the study of tumor development. In addition, as

27 only a small number of PDA patients are eligible for surgical intervention, the amount of human

28 tumor tissue available for research is limited. Therefore, methods to culture and/or propagate

29 tumor tissue are valuable as they offer researchers a way to probe cellular pathways involved in

30 tumorigenesis or to ascertain the effects of therapeutics. Traditional model systems such as

31 genetically engineered mice, cell lines, and xenografts have all led to powerful insights into PDA

32 tumorigenesis. More recently, researchers have turned to three-dimensional (3D), organotypic

33 culture methods, which hold great promise to circumvent some of the limitations of traditional

34 models. Advances in the field of regenerative medicine have led to the development of organoids

35 as 3D models. Recently, these developments came together, with the report of four new methods 
36 for modeling pancreatic cancer using organoids [4-7] (Figure 1). Here, we discuss traditional

37 methods for modeling PDA as well as these new organoid approaches. We also describe the

38 challenges and opportunities for using PDA organoids for clinical care.

Monolayer Cell Lines

41 Monolayer neoplastic cell lines have been a mainstay of cancer research. The first human cancer

42 cell line was generated from the cervical tumor of Henrietta Lacks and therefore named HeLa

43 cells [8]. Eleven years later, the first PDA cell line was reported [9]. Since that time, numerous

44 cell lines have been derived and represent tractable models for many different types of human

45 cancer. Once adapted to growth in serum-containing media in tissue culture dishes, cancer cell

46 lines are generally easy to culture, and can be passaged, cryopreserved and manipulated both

47 genetically and chemically.

49 While the use of monolayer cell lines in the study of cancer biology has led to numerous insights,

50 cell lines suffer from several limitations. Many tumors, especially slower growing, non-

51 metastatic lesions, fail to generate cell lines, with prostate cancer being a prime example [10].

52 For pancreatic cancer, the efficiency of generating cell lines from a resected primary tumor is

53 lower than the efficiency of generating 3D cultures [5]. Moreover, since most cell lines are

54 derived from a resected tumor specimen, and most PDA patients are ineligible for surgical

55 resection, PDA cell lines can only be generated for a small subset of patients and therefore do not

56 represent the diversity found across PDA tumors. In addition, neoplastic cell lines fail to

57 recapitulate key features of tumor biology, such as interactions with stromal cells and 
extracellular matrix (ECM). Furthermore, neoplastic cellular polarity, cell-cell contact, and 3D

59 organization are difficult to model when cells are cultured as monolayers [11-13].

61 The adaptation of neoplastic cells to monolayer tissue culture conditions has been shown to

62 fundamentally alter cells, selecting for clones that no longer model their primary counterparts

63 [14]. Culturing cells as monolayers in the presence of serum has been shown to promote the loss

64 of p53 function and to drive genomic instability $[5,14,15]$. Numerous studies have identified

65 gene expression differences between monolayer cell lines and their corresponding primary

66 tumors [12, 15-18], including comparisons of pancreatic cancer cell lines to primary PDA [18,

67 19]. These differences remain even when the presence of stromal cells in the primary tumor is

68 taken into account $[12,18]$. Thus, while there are many advantages to working with cancer cell

69 lines, they may not accurately model many aspects of tumor biology.

\section{Reprogrammed Monolayer Cultures}

72 As an alternative to standard, monolayer PDA cell lines, two recent publications report methods

73 that involve the addition of factors to support monolayer cultures. Kenneth Zaret and colleagues

74 isolated epithelial cells from a resected human PDA tumor and then induced expression of the

75 Oct4, Sox2, Klf4, and c-Myc transcription factors to reprogram the cancer cells [20]. These

76 reprogrammed cells required sustained expression of the transcription factors to remain

77 undifferentiated and were therefore called "induced pluripotent stem (iPS)-like" cells to

78 differentiate them from other iPS cells, which do not require sustained expression of the

79 reprogramming transcription factors. When the PDA-derived iPS-like cells were transplanted

80 subcutaneously into immunocompromised mice, they gave rise to structures that resembled 
81 pancreatic intraepithelial neoplasms (PanINs), pancreatic lesions believed to be the precursors of

82 PDA [20]. These results suggest that cellular reprogramming may partially suppress the cancer

83 phenotype, and this technique allows for a model of the earliest stages of tumorigenesis. To date,

84 this method has only been reported to successfully allow culture of one human tumor, and the

85 relatively low efficiency of colony formation suggests that clonal selection may be a potential

86 limitation of this technique. However, if the robustness of the method can be demonstrated, it

87 could be a useful approach for cultivating tumor cells from many patients.

89 Richard Schlegel and colleagues have developed an alternative approach to culturing primary

90 human tumor cells in which the cells are propagated on top of a confluent layer of irradiated

91 fibroblasts in the presence of an inhibitor of Rho-associated, coiled-coil-containing protein

92 kinase (ROCK), generating cultures which the authors call "conditionally reprogrammed cells"

93 [21]. ROCK signals downstream of the Rho GTPase pathway, and is involved in focal adhesion

94 formation, cell migration, stress fiber formation and regulation of apoptosis [22]. Inhibition of

95 ROCK promotes cell survival following dissociation [23]. How the irradiated fibroblasts support

96 growth is not entirely clear, but it likely involves secreted factors, since conditioned media from

97 irradiated fibroblasts can also promote the growth and survival of the tumor cells [21]. While

98 these approaches hold promise as new PDA models, the lack of 3D architecture in these cultures

99 remains a limitation. In addition, whether these culture conditions select for a subset of tumor

100 cells remains unclear.

101

102 Genetically Engineered Mouse Models 
103 The development of technologies to genetically engineer mice with specific mutations has led to

104 the generation of numerous murine models of PDA. (For a review, see [24]). Many of these

105 models use pancreas-specific $C r e$ drivers such as $P d x I$ - or P48-Cre, to activate oncogenic alleles

106 of Kras, a gene mutated in more than $90 \%$ of human PDA tumors [3]. Tumors arise

107 spontaneously in these mice, allowing early tumorigenesis to be studied. In addition, tumors

108 develop in 3D, and contain the dense desmoplasia and poor vasculature characteristic of human

109 PDA [25].

111 Genetically engineered mouse models offer an advantage over many of the other methods to

112 study PDA in that these models have native interactions between the neoplastic and stromal

113 cells. Thus, these models allow researchers to study interactions between tumor cells and the

114 native immune system and to explore the effects of immunotherapies [26]. In addition studies

115 using genetically engineered mice have revealed the complex interplay between PDA and

116 stromal cells, showing that cancer associated fibroblasts can play a tumor supportive [27, 28] or

117 tumor suppressive role [29, 30], depending on the context. Murine models have led to numerous

118 clinical insights into PDA, such as the discovery that albumin-coated paclitaxel could augment

119 the potency of gemcitabine by decreasing levels of cytidine deaminase, an enzyme responsible

120 for gemcitabine metabolism [31].

122 Despite the benefits of using engineered mice to study PDA, rapid experimental interrogation is

123 difficult with these models. Mouse models are time-consuming and expensive to breed, and the

124 engineering of additional mutations requires considerable effort. Furthermore, there have been

125 discoveries in murine models that have not been reproduced in the human setting [25, 30]. These 
126 may have been the result of interspecies differences, such as differences in drug metabolism [32],

127 immune function [33], and telomerase activity [34] between mice and humans. In addition, in

128 some murine PDA models, the oncogenic alleles are expressed during development, and murine

129 tumors are smaller and arise from fewer cell divisions than human tumors [35]. Finally, murine

130 models of PDA tend to have fewer mutations and less genetic complexity than human tumors

131 [36]. Taken together, murine models are powerful tools for interrogating PDA biology, but

132 scientists planning or interpreting studies involving murine models should be cognizant of these

133 limitations.

\section{Xenograft Approaches}

136 Alternative methods to model PDA involve the engraftment of human cell lines (cell-line-

137 derived xenografts, or CDXs) or human tumor fragments (patient-derived xenografts or PDXs)

138 into immunocompromised mice. CDXs are generated from cultured cell lines and therefore

139 suffer from many of the limitations described for monolayer cell lines, including selection during

140 culturing. In addition, while some have reported that xenograft tumors generated by

141 subcutaneous injection of cancer cell lines share histological characteristics with their

142 corresponding primary tumors [37], others have found that these tumors fail to recapitulate

143 primary tumor histology $[14,38]$. Furthermore, activity of therapeutic agents on CDX models

144 was found to have poor correlation with activity in human patients [39, 40]. This low correlation

145 held true for numerous studies using PDA xenografts (reviewed more extensively in [41]), and

146 may be attributable to the dense desmoplastic stroma and low blood vessel density found in

147 human PDA, which are not recapitulated in CDX's of this malignancy [25]. 
149 In contrast to CDXs, PDXs retain many of the characteristics of their corresponding primary

150 tumors, including tissue architecture and genomic alterations [42-45]. While subcutaneous

151 engraftment is commonly used to establish PDXs from human PDA, resemblance to primary

152 tumor was best when PDXs were established through transplantation into the pancreas

153 (orthotopically) [46, 47]. These results suggest that while orthotopic transplants are more

154 cumbersome to perform, they may generate more reliable data. Successful engraftment of PDA

155 tumors was shown to be significantly correlated with shorter survival [48], suggesting that highly

156 aggressive tumors are more amenable to PDX generation. PDXs have been used to find

157 mechanisms of resistance to targeted therapies and biomarkers to predict therapeutic response

158 [44]. However, following engraftment, PDXs derived from PDA become infiltrated with murine

159 stroma [43], and most human stroma is replaced with murine cells long before the fourth or fifth

160 passage which is when PDXs are most likely to be used for clinical testing [42]. The mismatch

161 between human tumor cells and mouse stroma should be considered when evaluating studies

162 involving PDXs.

164 Overall, studies have found that clinical response of PDXs to therapeutics is correlated with 165 response in patients (reviewed in [42]). However, only limited studies have been done to look at 166 the clinical response of PDXs generated from PDA patients. Manuel Hidalgo and colleagues 167 compared the clinical response of 23 patients and their corresponding PDXs when treated with 168 gemcitabine, and found that in 7 of the 23 pairs, the sensitivity of the PDXs to gemcitabine 169 correlated with a longer median time to progression in the patient [48]. A separate study from 170 this group subjected 4 PDA-derived PDXs to 63 different therapies in 232 combinations to 171 determine the most effective agents against each PDX [49]. While 3 of the 4 PDXs were most 
172 sensitive to gemcitabine, which is already a standard of care for PDA, 1 PDX showed sensitivity

173 to mitomycin $\mathrm{C}$ and cisplatin. When the patient matching this PDX progressed on gemcitabine,

174 he was treated with mitomycin $\mathrm{C}$ and later with cisplatin, and was reported to be disease-free 50

175 months after his initial diagnosis [49]. These results suggest that PDXs may have predictive

176 value in the clinic, but further studies are needed to determine how well they correlate with the

177 response of PDA patients and to better understand the reasons why responses of some PDXs fail

178 to correlate with those of patients.

180 While initial studies using PDX models are promising, this model has a number of limitations

181 that may prevent its widespread use. Relatively large amounts of resected tissue or biopsy

182 material are needed to generate PDX models, limiting the applicability of this model to most

183 PDA patients. Moreover, PDXs take 4-8 months to produce and require lengthy passaging to

184 generate experimental cohorts [50]. The generation and maintenance of large numbers of

185 immunocompromised mice to passage the PDXs can be costly. Finally, the use of

186 immunocompromised mice limits the use of PDXs to study immunotherapies. Nonetheless, the

187 PDX method allows for the propagation and study of many types of tumors in an in vivo setting.

189 Three Dimensional Models of Pancreatic Tumorigenesis

190 The limitations in the systems available to study pancreatic tumorigenesis prompted researchers

191 to find novel ways to model PDA. To that end, 3D culture methods hold promise as systems to

192 better mimic the biology found in vivo. A key goal of 3D culture methods is to prevent cells from

193 attaching to the bottom of the culture dish, either by keeping cells in suspension or by culturing

194 cells in the presence of a matrix such as collagen or Matrigel (Box 1). 3D methods to culture 
195 pancreas cells are hardly a new phenomenon. Rather, they have emerged as part of a larger

196 movement over the last century to be able to culture and study tissues ex vivo.

198 The Development of Three-Dimensional Approaches to Culturing Pancreas Cells

199 Researchers have been attempting to culture the pancreas outside the body as far back as 1938 ,

200 when Carrel and Lindberg were able to keep a cat pancreas alive in culture for 4 days using a

201 perfusion pump [51]. By the 1980s, numerous methods for culturing the pancreas as organ

202 explants had been reported and researchers were starting to develop methods for culturing

203 isolated pancreas cells in 3D [52]. One early study found that primary rat pancreatic duct cells

204 cultured in agarose formed closed lumina and could be maintained in culture up to 6 weeks [53].

205 At this time, primary culture methods were employed to aid in the study of pancreatic endocrine

206 cells. For instance, when cultured in the presence of collagen, dissociated rat pancreas cells were

207 reported to associate into "islet-like organoids" [54]. Human pancreas cells embedded in

208 Matrigel or in rat tail collagen were found to proliferate and form cysts that expressed pancreatic

209 ductal markers as well as structures resembling endocrine islets [55]. While these early methods

210 did not allow for the passage of pancreatic cells in culture, they did provide valuable insights into

211 pancreatic development and endocrine function.

213 Building off the successes of early explant cultures, researchers have developed methods for

214 organotypic culture of pancreatic tissue slices. Speier and colleagues were able to successfully

215 culture murine pancreas tissue slices embedded in agarose for up to 7 days [56]. Similar methods

216 were used to culture human normal pancreas tissue and resected PDA tumor specimens as

217 organotypic slice cultures, which were viable for up to 6 days and amenable to transduction with 
218 adenoviral vectors [57]. By using a precision vibratome and slicing tissue immediately following

219 surgical resection, Loda and colleagues were able to culture 300-500 $\mu \mathrm{M}$ slices of colon, lung,

220 and prostate carcinomas without needing to embed in agarose [58]. This technique was used to

221 culture resected human PDA tumors and to evaluate the effects of mitogen-activated kinase

222 (MEK) and phosphoinositide 3-kinase (PI3K) inhibitors [59]. Organotypic slice cultures retain

223 stromal cells and preserve tumor-stroma interactions, which could be a major benefit to these

224 cultures. However, depending on the size of the slice, diffusion of compounds or oxygen may be

225 limited in these cultures. In addition, organotypic slice cultures can only be maintained for up to

226 a week and do not rapidly proliferate in culture, so the amount of material that can be generated

227 from a single patient is limited.

229 Methods have also been developed to allow for limited culture of partially dissociated primary

230 normal and neoplastic pancreas tissue, leading to advances in our understanding of pancreatic

231 tumorigenesis. For instance, the Rustgi Laboratory developed a method to culture pancreatic

232 ducts for up to 10 months embedded in collagen [60]. The Bar-Sagi Laboratory developed

233 methods for isolating and culturing primary murine pancreatic duct cells on top of a laminin-

234 coated coverslip, embedded in high-concentration of Matrigel, or as an overlay in low-

235 concentration Matrigel on top of a Matrigel bed [61]. By isolating pancreatic duct cells from a

236 mouse with the $\operatorname{Kras}^{L S L-G 12 D}$ allele and exposing the cells to Cre, the researchers were able to

237 study how oncogenic Kras prevents cellular senescence [62]. Immortalized human duct cell lines

238 embedded in Matrigel formed invasive, disorganized structures only in the presence of the

$239 \operatorname{Kras}^{G 12 D}$ oncogene, a finding that was only revealed by 3D culture [63]. Murine tumor cells or 
240 human PDA cell lines cultured in Matrigel on top of a bed of agar could be treated with

241 chemotherapies and chemical inhibitors to assay for drug sensitivity [64].

243 In addition to these primary culture systems, methods to culture human PDA monolayer cell

244 lines in 3D were developed. In one early study, cells from a PDA cell line were cultured under

245 continuous agitation, which prompted the cells to self-organize into polarized spherical structures

246 called "spheroids" [65]. Since then, numerous methods have been reported. Some generate PDA

247 spheroids by preventing cellular attachment through the use of special culture devices [66], low-

248 attachment or agarose coatings [67-69], mechanical stirring [70], or by only passaging floating

249 spheres within the culture well [71]. Others culture cells on top of or embedded inside of

250 matrices such as fibronectin, methylcellulose, type I collagen, or Matrigel (Box 1) [11, 72-75]. A

251 recent study reported the culture of a human PDA cell line inside synthetic scaffolds made of

252 biocompatible polymers [76]. Finally, methods to co-culture PDA cells with stromal cells in 3D

253 have also been described [77, 78]. Unlike the monolayer cell lines from which they are derived,

254 spheroid cultures have been shown to take on characteristics more similar to those of cells in

255 vivo, including the production of ECM, increased resistance to therapies, and the appearance of

256 polarized cell junctions $[68,69,75]$. However, as described in the previous section, the use of

257 monolayer cell lines as a starting point limits the in vivo relevance of these approaches.

259 While some use spheroid cultures to mimic 3D interactions found in vivo, others use spheroids as

260 an assay for cells capable of self-renewal. In the latter case, cells must be seeded for spheroid

261 formation at a low density, ensuring that the outgrowing spheroids were derived from

262 propagating cells [79]. Such methods have been used to enrich for cells in the pancreas with the 
263 properties of adult stem cells, including the capacity to self-renew and the ability to differentiate

264 into multiple lineages [80, 81]. Similarly, spheroids derived from PDA cell lines seeded at a low

265 density have been used to enrich for cells with the characteristics of cancer stem cells [79]. Such

266 spheroids have been shown to have enhanced capacity to form tumors following transplant and

267 enrichment of known stem cell markers such as CD44 and CD133 [69, 71, 82]. However, the use

268 of spheroid models as an assay for cancer stem cells should be interpreted carefully: while

269 spheroids assay for the capacity of an isolated set of cells to self-renew under the culture

270 conditions, it remains unclear how such cells correspond to tumor cells in vivo [83]. In addition,

271 variation in spheroid methods, the propensity for spheroids to aggregate in culture, and the

272 potential for quiescent tumor stem cells not to proliferate under spheroid conditions all may

273 influence the reliability of these assays [83]. Nonetheless, the clonogenic sphere-forming assay

274 remains a powerful technique to enrich for cells with stem-like properties.

275

276 Organoid Cultures

277 As methods for culturing PDA cells in 3D were increasing in popularity, researchers in other

278 fields were working out methods to culture primary normal tissue and later, primary tumor cells

279 as "organoids." Organoid models have been described for the stomach [84-86], small intestine

280 [87-89], colon [87, 89], liver [90, 91], and mammary gland [92], as well as numerous other

281 tissues [93]. In addition, tumor organoid models have been developed for breast [92], colon [89],

282 and prostate [10] cancers. More recently, pancreas and pancreatic cancer organoids have been

283 developed [4-7, 94, 95].

284 
285 Currently, no standard definition of "organoid" exists. The term "organoid" literally means

286 organ-like, reflecting the ability of organoid culture conditions to prompt cells to self-organize

287 into structures mimicking the architecture of the organ from which they were derived. While

288 some have suggested that the term "organoid" should be reserved for cultures in which more

289 than one cell type is present, as in an intact organ [4], this definition has not been universally

290 adopted. Nonetheless, different organoid models generally share a couple of characteristics. As

291 with many other 3D culture methods, organoids are cultured on top of or embedded inside of a

292 matrix (Box 1). In contrast to organ explant or organotypic slice cultures, where a large piece of

293 tissue is cultured as an intact unit, organoids are generated from smaller multicellular units (such

294 as enzymatically or mechanically dissociated tissue) or from single cells. Organoids share many

295 similarities with spheroid cultures, and the terms have often been used interchangeably in the

296 literature. For clarity, we suggest that 3D cultures that are generated from cells initially cultured

297 as monolayers fall under the purview of "spheroid" cultures. In contrast, the term "organoid"

298 should be reserved for 3D cultures that are generated from dissociated primary tissue or from

299 embryonic stem or iPS cells differentiated in vitro, and are propagated using a matrix and a

300 media that promotes cell proliferation.

302 Non-neoplastic organoids are thought to arise from adult stem cells, and therefore should be 303 capable of self-renewal and differentiation [96]. Consistent with this idea, flow-sorted pancreatic 304 duct cells that stained positively for known progenitor/stem cell markers were more efficient at

305 forming organoids than the general pancreatic duct cell population and much more efficient than

306 progenitor-depleted duct cells [97]. The capacity for differentiation can be determined by

307 transplanting the organoid into a mouse and demonstrating that the organoid can differentiate 
308 into structures resembling the tissue from which the organoid was derived. However, not all

309 organoid systems have demonstrated both self-renewal and differentiation capabilities. In

310 addition, whether tumor-derived organoids result from the outgrowth of a cancer stem cell

311 population remains unclear.

313 The laboratories of Anne Grapin-Botton and Hans Clevers have both developed methods for

314 culturing normal murine pancreata in Matrigel as organoids [94, 95]. Grapin-Botton and

315 colleagues cultured murine embryonic pancreas cells inside Matrigel in order to study pancreatic

316 duct development [94]. These embryonic pancreas progenitor organoids proliferated in culture

317 and underwent branching morphogenesis. In contrast, the Clevers group, building on their prior

318 work [86, 88, 89, 91], developed methods to propagate adult murine pancreatic duct cells as

319 organoids [95]. When embedded in serum-free Matrigel and provided a defined, cocktail of

320 growth factors, including the Lgr5 agonist R-spondin I to stimulate Wnt signaling, pancreatic

321 duct cells formed proliferating, cystic spheres. Flow-sorting experiments have demonstrated that

322 these conditions allow the propagation of pancreatic duct but not acinar or islet cells [97]. While

323 these organoids retained ductal characteristics in culture, they could form both duct-like,

324 Cytokeratin 19-positive structures as well as endocrine-like, Insulin-positive cells following

325 transplantation into the mouse renal capsule, demonstrating that cells cultured with this method

326 are bipotent [95].

328 In collaboration with the Tuveson Laboratory, the Clevers group extended this work to the

329 culture of neoplastic pancreas cells [5]. Cells taken from PDA tumors and metastases that formed

330 in genetically engineered mouse models could be cultured under identical conditions to normal 
331 pancreatic duct cells. Cells isolated from PanIN lesions that arise in genetically engineered

332 mouse models could also be cultured as organoids. These organoids can be cryopreserved and

333 passaged indefinitely, and are amenable to genetic, transcriptomic, proteomic, and biochemical

334 analyses. Thus, this system is ideal for probing changes involved at each stage in tumorigenesis.

336 With some modifications to the culture media, including the addition of Wnt3a, normal and

337 neoplastic human organoids could be cultured. As with the murine organoids, human organoids

338 proliferated readily under these conditions and could survive cryopreservation. While under

339 current culture conditions, human normal organoids could only be cultured for 20-25 passages,

340 human PDA organoids could be passaged indefinitely. Unlike the murine organoids, human PDA

341 organoids took on a range of morphologies, often proliferating as filled spheres. This method

342 worked well even with low numbers of starting cells, allowing for the generation of human PDA

343 organoids from fine needle aspirate (FNA) biopsies and dramatically expanding the types of

344 tumors capable of being studied in the laboratory. Like the murine organoids, organoids

345 generating using this method are amenable to genetic and biochemical analysis: a recent study

346 found that human patient-derived PDA organoids were sensitive to inhibition of MAPK-

347 interacting protein kinase (MNK) with CGP57380 [98], showing that organoids can be used to 348 study drug sensitivity.

350 In contrast to transplants of monolayer cell lines which rapidly form aggressive adenocarcinomas

351 [25], orthotopic transplants of these murine or human PDA organoids initially give rise to

352 structures resembling PanIN which only later progress to frank PDA resembling the tumor of

353 origin [5]. A number of reasons could explain this transplant phenotype. The organoid culture 
354 system may "reprogram" more aggressive cancer cells to a less aggressive state. Alternatively,

355 organoid culture may select for less aggressive cancer cells more likely to give rise to PanIN

356 when transplanted. Monolayer cultures may also select for more aggressive cancer cells, which

357 can readily form tumors following transplantation. It is also possible that less aggressive

358 subclones present in the organoid cultures or normal cells present in the host mouse have a

359 "neighbor suppressive" effect on PDA formation initially after transplant of the organoids. In

360 line with this idea, in vitro studies using monolayer cultures have shown that fibroblasts can

361 inhibit the growth of transformed cells [99], although the role of neighbor suppression in PDA

362 remains unclear. More studies are needed to determine the biological basis for this transplant

363 phenotype, and the relatively long time (2-8 months depending on the organoid culture) that

364 transplanted tumors take to form frank PDA may be a drawback to this system. Nonetheless, the

365 ability of PDA organoids to form PanIN-like structures following transplant can be exploited to

366 study early pancreas cancer, which may aid in the discovery of new diagnostic tools. In addition,

367 murine organoids derived from normal duct cells as well as PanINs, PDAs, and metastases

368 provide a progression model to examine changes associated with each stage of pancreatic

369 tumorigenesis.

371 Melissa Skala's Laboratory recently described a similar method for culturing murine and human

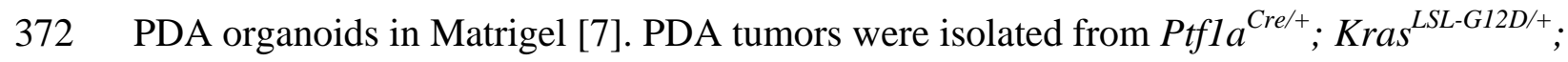

$373 T g f b r 2^{f l / f 1}$ mice, digested, and embedded in a 50\%/50\% mix of Matrigel and serum-containing

374 culture media. Under these conditions, both fibroblasts and cytokeratin-positive tumor cells were

375 able to propagate, with the latter propagating as both spherical and asymmetric organoids.

376 Organoids cultured under these conditions were amenable to optical metabolic imaging (OMI), 
377 which can be used to quantify levels of NADPH and FAD as a proxy for cellular redox state and

378 overall cell health. OMI was used to probe the response of murine PDA organoids to

379 gemcitabine as well as to inhibitors of Janus kinase (JAK, AZD1480), MEK (AZD6244), or

380 PI3K (XL147). Using this method, the authors were also able to generate organoids from a

381 resected human PDA tumor [7]. Unlike the murine organoids, the human PDA organoids took on

382 many morphologies. OMI revealed that the human PDA organoids were relatively insensitive to

383 the JAK inhibitor, but showed some sensitivity to gemcitabine, or to the combination of

384 gemcitabine with the JAK inhibitor. While relatively little characterization of these organoids

385 has been reported, and the significance of the multiple organoid morphologies remains unclear,

386 the OMI method may be of interest for those wishing to perform drug screens in 3D.

388 Senthil Muthuswamy and colleagues recently reported a third method for generating pancreatic

389 organoid cultures [6]. Human pluripotent stem cells were converted to pancreatic progenitor cells

390 in vitro, and cultured as an overlay in media on top of a Matrigel bed. Under these conditions,

391 the progenitors organized into cystic spheres which secreted an outer layer of basement

392 membrane and maintained gene expression patterns similar to those of pancreatic progenitor

393 cells. Addition of exogenous ligands to stimulate Wnt signaling was not needed to maintain these

394 cultures. Consistent with their progenitor state, these organoids were able to form both acinar and 395 ductal cells when exposed to differentiation signals.

397 The Muthuswamy group extended this method to generate organoid cultures from surgically

398 resected human PDA tumors with high efficiency [6]. Human PDA organoids showed dysplastic

399 morphologies and often propagated as filled spheres. Organoids could be passaged and survived 
cryopreservation. Organoids subcutaneously transplanted into mice formed frank

401 adenocarcinomas that resembled their tumors of origin. Intriguingly, a single culture of human

402 PDA organoids could show multiple diverse histologies, reminiscent of the multiple histologies

403 seen in the primary tumor, suggesting that this culture system may be capturing the intratumoral

404 heterogeneity found in primary tumors. It is not clear whether untransformed human pancreatic

405 tissue can be propagated using this organoid technique, which may limit experiments requiring

406 an untransformed control generated under the same conditions. However, these organoids were

407 amenable to small-scale therapeutic screening: the authors tested the growth of organoids in the

408 presence of epigenetic pathway inhibitors and identified a subset of human organoids sensitive to

409 inhibition of Enhancer of zeste homolog 2 (EZH2) [6]. The potential to use patient-derived

410 organoids for therapeutic screening could have great clinical benefit.

412 Finally, Calvin Kuo and colleagues developed a distinct method for culturing pancreas cells as

413 organoids [4], which built off their prior work culturing murine intestinal organoids [87]. The

414 authors use an "air-liquid interface" (ALI) method in which embryonic tissue fragments are

415 cultured in a type I collagen gel that rests on a permeable substrate with media underneath,

416 allowing nutrients to diffuse from the bottom. The top of the culture is exposed to air, thus

417 allowing cells to receive higher levels of oxygen than would be encountered in traditional culture

418 methods and preventing hypoxic death. When grown as ALI cultures, murine neonatal pancreatic

419 tissue formed cystic organoids consisting mainly of E-cadherin- and Pdx1-positive ductal

420 epithelial cells surrounded by alpha-SMA-positive stromal cells [4]. These cultures did not

421 require exogenous growth factor supplementation and were viable for up to 50 days, but could

422 not be passaged. 
424 Next, the authors generated ALI pancreatic organoids from mice bearing the $\mathrm{Kras}^{L S L-G 12 D /+}$ or

$425 \operatorname{Trp} 3^{f l f l}$ alleles [4]. Following infection with Adenoviral-Cre to activate oncogenic Kras or

426 delete p53, the ALI organoids were able to proliferate and could be serially passaged [4]. Cre-

427 infected $\mathrm{Kras}^{\mathrm{LSL-G12D/+} \text { organoids, Trp53 } 3^{\text {flfl }} \text { organoids, or Kras }}{ }^{L S L-G 12 D /+} ; \operatorname{Trp}^{\text {fllfl }}$ double-mutant

428 organoids were all capable of forming tumors when transplanted subcutaneously. While it

429 remains unclear whether PDA tumor cells, which often face hypoxia in vivo, will propagate

430 under ALI conditions. However, the ability to model the effects of Kras mutation alone or in

431 combination with Trp53 mutation offers a promising method for studying the steps in

432 tumorigenesis. In addition, the presence of stromal cells in these cultures may better mimic

433 tumor-stroma interactions than models where epithelial cells are cultured alone.

\section{Using Organoids for Personalized Medicine}

436 The relatively rapid generation and high proliferation of organoid cultures makes them well

437 suited for therapeutic research. However, as of this review, there have only been limited

438 examples of therapeutic interrogation using pancreas organoids $[6,7,98]$. Currently, the most

439 comprehensive work in this domain utilizes the intestinal and colonic organoids, and could serve

440 as a model for future pancreas organoid research. Marc van de Wetering and colleagues

441 assembled an organoid biobank from 20 colorectal cancer patients [100]. They performed deep

442 genomic and transcriptomic analyses using both neoplastic and adjacent-normal organoids, thus

443 providing meaningful comparison and identification of tumor-specific DNA and RNA variations.

444 The tumor organoids were screened in a high-throughput manner using a custom library of

445 therapeutic compounds to identify compounds the organoids were sensitive to. This approach led 
446 to the identification of effective patient-specific treatments. There was a correlation between

447 therapeutic response and mutational status, confirming previously known, mutation-based drug

448 sensitivities. Importantly, some therapeutic responses could not have been predicted through

449 sequencing analysis alone, highlighting the value of such an approach. To further determine the

450 predictive value of personalized organoid screening, a observational colorectal cancer trial is

451 now underway at the Netherlands Cancer Institute in Amsterdam [101].

453 An organoid-based personalized medicine approach is also being investigated for the diagnosis

454 and treatment of cystic fibrosis (CF). This clinical advance stems from the work of Johanna

455 Dekkers and colleagues who cultured primary intestinal organoids from CF patients [102]. Due

456 to an inactivating mutation in the Cystic fibrosis transmembrane conductance regulator (CFTR)

457 gene, the organoids demonstrate poor transmembrane conductance in culture in response to

458 forskolin-induced high intracellular cyclic AMP content, thereby recapitulating a hallmark of

459 cystic fibrosis in a dish. Gerald Schwank and colleagues were able to apply CRISPR/Cas9

460 genome editing tools to repair the mutant CFTR locus in organoid cultures, thus rescuing the CF

461 phenotype [103]. In addition, this defect could be rescued through drug treatment in these ex vivo

462 culture models. Distinct CFTR mutant organoids responded differently to treatments, and

463 surprisingly, even organoids with the same CFTR mutation displayed differential treatment

464 responses suggesting that organoids may be used to quickly screen and identify the best patient-

465 specific-treatment [102]. If translated to the clinic, such a personalized approach may help CF

466 patients quickly identify therapies they are most likely to benefit from. 
468 The ability to rapidly and efficiently genetically engineer organoids using the CRISPR/Cas9

469 system also enables cancer research. Two laboratories independently generated isogenic human

470 intestinal organoids lines harboring both tumor suppressor and oncogenic mutations [104, 105].

471 Using these genetically engineered human organoids, researchers were able to study tumor

472 development and invasive potential. The ability to engineer sequential mutations in human

473 pancreas organoids could aid in the generation of new models based on the newly identified 474 subtypes of the PDA [106].

475

476 Bolstered by the results of these preliminary studies, we expect pancreas organoids to advance

477 the state of personalized medicine for PDA. Organoids offer an ideal setting for patient-specific

478 assays as genetic traits of disease such as driver mutations and chromosomal copy number are 479 preserved [10, 107]. The diagnosis of pancreatic cancer often involves collection of a biopsy, 480 such as an endoscopic ultrasound-guided FNA. Unlike many other culture systems, organoids 481 may be cultured from just one needle pass with a high success rate [5]. This enables the 482 possibility of deep genomic characterization and ex vivo therapeutic testing in PDA patients that 483 have traditionally been under-studied in research settings. It will be important to determine if 484 these biopsy-derived organoid cultures faithfully represent the genetic heterogeneity and 485 therapeutic sensitivity profile of the entire primary tumor. Conversely, in patients with resectable 486 disease, multiple distinct organoid lines could be generated from cross-sections of the same 487 tumor, and used to model the intratumoral clonal heterogeneity found in PDA patients [108]. 488 Once established, PDA organoid cultures could be expanded, genotyped, and challenged with 489 approved, standard of care therapies within weeks, possibly allowing for organoid-guided, 490 second-line therapeutic options being offered to the patient. A similar approach tested in human 
491 liver organoids yielded promising results indicating that the organoid system was amenable to

492 therapeutic testing [109]. Methods are being developed for drug discovery using high throughput

493 testing in 3D cultures which could be applied to therapeutic testing in patient-derived organoids

494 [110]. Finally, a retrospective study using prostate organoids found that the organoids derived

495 from distinct patients displayed different responses to therapies which correlated with the

496 observed genetics of each patient's cancer [10], suggesting that therapeutic testing of organoids

497 will have clinical benefit.

499 A critical first step for the use of organoids for personalized approaches to PDA care will be an 500 efficacy evaluation within a retrospective clinical trial. In one possible clinical trial, patients with 501 advanced pancreatic cancer would consent to donate a portion of biopsy tissue for the purposes 502 of establishing organoids. Patient-derived organoids would then be used to test therapeutic 503 sensitivities against standard of care drugs, and the results of these assays would be 504 retrospectively compared with the patient's tumor response to therapy. A good correlation 505 between tumor response/progression and organoid sensitivity/resistance to a particular

506 therapeutic regimen would indicate that the organoid system is predictive of treatment outcome. 507 If such a study were successful for PDA, then a prospective study could be undertaken with the 508 goal of identifying optimal treatments to directly improve patient survival and quality of life.

509 Such studies could also be used to identify second-line treatments following the development of 510 resistance to first-line therapies.

512 There are numerous challenges to implementing these studies in the clinic. Considering the short 513 median survival of pancreatic cancer patients, the main hurdle to this approach will be to reduce 
514 the time necessary to culture and assess the organoids as to offer insight to the clinical care team

515 as soon as possible. Currently, heavy pre-treatment with chemotherapy prior to surgery/biopsy,

516 and low neoplastic cellularity of biopsy tissue are the major factors for failed organoid cultures,

517 which may pose a problem for studying such patients. Optimization of culture methods to more

518 quickly and efficiently generate organoids from such samples may enable sampling of a broader

519 spectrum of patients. A second challenge is the lack of immune, stromal, and vasculature

520 compartments in the organoid culture, thus preventing the effective testing of immuno-therapy

521 (e.g. immune checkpoint inhibitors) or stroma-modulating drugs (e.g. Vitamin D analogues). It

522 will be important for future studies to develop and optimize co-culturing methods to generate a

523 more complete and representative ex vivo system.

524

525 Before clinical studies using organoids are undertaken, efforts should be made to better

526 characterize PDA organoids. The different organoid methodologies should be compared directly,

527 and protocols for isolation and culturing of PDA organoids should be standardized. Ideally,

528 components involved in the culture matrix and medium would also be standardized. Most current

529 approaches use Matrigel, which has large variation between lots. Synthetic matrices consisting of

530 fully defined components may help to circumvent this issue. These optimization efforts are very

531 important, as human organoid culture is currently an expensive and technically challenging

532 method requiring precious patient samples. Finally, organoid models should be compared to cell

533 line and xenograft approaches to determine the optimal uses for each model.

534

535 Concluding Remarks 
536 While there are many questions about organoid cultures that still need to be addressed (see

537 Outstanding Questions), these 3D culture methods have emerged as powerful systems for

538 probing PDA biology. Such methods offer the ability to generate a self-renewing source of

539 patient-specific cells that is amenable to genetic and chemical perturbations. This will allow for

540 basic discoveries into the pathways driving different PDA tumors and for the identification of

541 therapies for PDA. Moreover, organoids are likely to be increasingly incorporated in clinical

542 approaches to PDA care, where they may provide great benefit in guiding individual treatment

543 decisions. 


\section{ACKNOWLEDGEMENTS}

545 We apologize to our colleagues whose work could not be cited due to space constraints. We are

546 thankful to members of the Tuveson Laboratory for helpful discussions and to Sylvia Boj for her

547 critical reading of this review. L.A.B was supported by the National Cancer Institute of the

548 National Institutes of Health under Award Number F32CA192904. D.A.T. is a distinguished

549 scholar of the Lustgarten Foundation and Director of the Lustgarten Foundation-Designated

550 Laboratory of Pancreatic Cancer Research. D.A.T. is also supported by the Cold Spring Harbor

551 Laboratory Association, the Carcinoid Foundation, PCUK, and the David Rubinstein Center for

552 Pancreatic Cancer Research at MSKCC. In addition, we are grateful for support from the

553 following: Stand Up to Cancer/KWF (D.A.T.), the STARR foundation (I7-A718 for D.A.T.),

554 DOD (W81XWH-13-PRCRP-IA for D.A.T.), PMRA (H.T. and D.A.T), and the NIH

555 (5P30CA45508-26, 5P50CA101955-07, 1U10CA180944-01, 5U01CA168409-3, and

556 1R01CA190092-01 for D.A.T.). 


\section{LEGENDS}

559 KEY FIGURE

560 Organoid models of pancreatic ductal adenocarcinoma

\section{Figure 1. Organoid models of pancreatic ductal adenocarcinoma}

562 Diagrams of a cross-section of a single organoid culture well for each of the four recently

563 reported methods for modeling PDA using organoids, the Clevers and Tuveson Laboratories

564 method [5], the Skala Laboratory method [7], the Muthuswamy Laboratory method [6], and the

565 Kuo Laboratory method [4]. Characteristics of each system are shown below diagrams. Matrix

566 components are depicted in blue, culture medium is in orange, and organoids are in yellow. The

567 targeted therapeutic study using organoids cultured with the Clevers and Tuveson organoid

568 method is described in Ref [98]. MNK: mitogen activated protein kinase-interacting protein

569 kinase. JAK2: Janus kinase 2. MEK: mitogen-activated protein kinase kinase. PI3K:

570 phosphoinositide 3-kinase. FNA: Fine needle aspirate. 


\section{TEXT BOX}

\section{Box 1. 3D Culture Matrices}

573 3D culture methods typically involve embedding cells inside of or culturing cells on top of a

574 matrix. Substances such as Matrigel, hyaluronic acid, laminin, collagen, polyethylene glycol

575 hydrogels, photoactivatable hydrogels, and peptide hydrogels have all been used as matrices for

576 3D culture $[111,112]$. These substances provide a physical barrier, preventing cells from

577 interacting with and attaching to the culture dish. In addition, the matrix acts as a physical

578 structure with which cells can interact. Depending on the matrix material, this structure provides

579 the cells with biomechanical and sometimes biochemical cues that mimic the environment of the

580 ECM in vivo [112]. Collagen and Matrigel are the two most common matrices used for 3D

581 culture of pancreas cells.

582

583 Collagen

584 There are at least 26 distinct types of collagen, of which types I and IV are most often used for

585 culture. Type I collagen is the most abundant collagen in the human body, and is found in

586 tendon, bone, skin, and other interstitial connective tissues [113]. This collagen can be purchased

587 either as recombinant protein or purified from rat tail. Type IV collagen is the primary

588 component of the basement membrane of normal tissue, including pancreatic epithelium [113-

589 115]. PDA progression is associated with breakdown of the basement membrane architecture and

590 with increased production of type I collagen as part of the desmoplastic reaction $[116,117]$.

591

592 Matrigel 
593 Matrigel is a commercial name for a basement membrane extract that is purified from murine

594 Engelbreth-Holm-Swarm (EHS) tumors [118]. Matrigel contains high levels of type IV collagen

595 as well as laminin and enactin/nidogen [118]. In addition, more than 1800 proteins have been

596 identified in Matrigel, including numerous growth factors such as epidermal growth factor

597 (EGF), basic fibroblast growth factor (bFGF), transforming growth factor beta (TGF- $\beta$ ), and

598 platelet-derived growth factor (PDGF) [119]. For researchers wishing to control growth factor

599 levels in their cultures, a growth factor-reduced preparation of Matrigel is available [120],

600 although levels of TGF- $\beta$ are not much reduced in this preparation, which may impact the

601 growth of some cell types [119]. When cultured in Matrigel, numerous cell types, including

602 pancreatic cells, have been shown to take on a polarized morphology and to have physiologic

603 responses not seen in cells cultured as monolayers [118].

604 
607 1. Siegel, R.L., K.D. Miller, and A. Jemal, Cancer statistics, 2015. CA: a cancer journal for $608 \quad$ clinicians, 2015. 65(1): p. 5-29.

2. Rahib, L., et al., Projecting cancer incidence and deaths to 2030: the unexpected burden of thyroid, liver, and pancreas cancers in the United States. Cancer research, 2014.

3. Ryan, D.P., T.S. Hong, and N. Bardeesy, Pancreatic adenocarcinoma. The New England

4. Li, X., et al., Oncogenic transformation of diverse gastrointestinal tissues in primary

5. Boj, S.F., et al., Organoid models of human and mouse ductal pancreatic cancer. Cell, 2015. 160(1-2): p. 324-38.

6. Huang, L., et al., Ductal pancreatic cancer modeling and drug screening using human pluripotent stem cell- and patient-derived tumor organoids. Nature medicine, 2015. 21(11): p. 1364-71.

10. Gao, D., et al., Organoid cultures derived from patients with advanced prostate cancer.

7. Walsh, A.J., et al., Optical Imaging of Drug-Induced Metabolism Changes in Murine and Human Pancreatic Cancer Organoids Reveals Heterogeneous Drug Response. Pancreas, 2015.

8. Gey, G.O., W.D. Coffman, and M.T. Kubicek, Tissue Culture Studies of the Proliferative Capacity of Cervical Carcinoma and Normal Epithelium. Cancer research, 1952. 12(4): p. 264-265.

9. Dobrynin, Y.V., Establishment and Characteristics of Cell Strains from Some Epithelial Tumors of Human Origin. Journal of the National Cancer Institute, 1963. 31(5): p. 1173$\&$.

11. Serebriiskii, I., et al., Fibroblast-derived 3D matrix differentially regulates the growth and drug-responsiveness of human cancer cells. Matrix biology : journal of the International Society for Matrix Biology, 2008. 27(6): p. 573-85.

12. Birgersdotter, A., R. Sandberg, and I. Ernberg, Gene expression perturbation in vitro--a growing case for three-dimensional (3D) culture systems. Seminars in cancer biology, 2005. 15(5): p. 405-12.

13. Edmondson, R., et al., Three-dimensional cell culture systems and their applications in drug discovery and cell-based biosensors. Assay and drug development technologies, 2014. 12(4): p. 207-18. 
14. Lee, J., et al., Tumor stem cells derived from glioblastomas cultured in bFGF and EGF more closely mirror the phenotype and genotype of primary tumors than do serumcultured cell lines. Cancer cell, 2006. 9(5): p. 391-403.

15. van Staveren, W.C., et al., Human cancer cell lines: Experimental models for cancer cells in situ? For cancer stem cells? Biochimica et biophysica acta, 2009. 1795(2): p. 92103.

16. Ertel, A., et al., Pathway-specific differences between tumor cell lines and normal and tumor tissue cells. Molecular cancer, 2006. 5(1): p. 55.

17. Sandberg, R. and I. Ernberg, Assessment of tumor characteristic gene expression in cell lines using a tissue similarity index (TSI). Proceedings of the National Academy of Sciences of the United States of America, 2005. 102(6): p. 2052-7.

18. Stein, W.D., et al., A Serial Analysis of Gene Expression (SAGE) database analysis of chemosensitivity: comparing solid tumors with cell lines and comparing solid tumors from different tissue origins. Cancer research, 2004. 64(8): p. 2805-16.

19. Gadaleta, E., et al., A global insight into a cancer transcriptional space using pancreatic data: importance, findings and flaws. Nucleic acids research, 2011. 39(18): p. 7900-7.

20. Kim, J., et al., An iPSC line from human pancreatic ductal adenocarcinoma undergoes early to invasive stages of pancreatic cancer progression. Cell reports, 2013. 3(6): p. 2088-99.

21. Liu, X., et al., ROCK inhibitor and feeder cells induce the conditional reprogramming of epithelial cells. The American journal of pathology, 2012. 180(2): p. 599-607.

22. Riento, K. and A.J. Ridley, Rocks: multifunctional kinases in cell behaviour. Nature reviews. Molecular cell biology, 2003. 4(6): p. 446-56.

23. Zhang, L., et al., ROCK inhibitor Y-27632 suppresses dissociation-induced apoptosis of murine prostate stem/progenitor cells and increases their cloning efficiency. PloS one, 2011. 6(3): p. e18271.

24. Perez-Mancera, P.A., et al., What we have learned about pancreatic cancer from mouse models. Gastroenterology, 2012. 142(5): p. 1079-92.

25. Olive, K.P., et al., Inhibition of Hedgehog signaling enhances delivery of chemotherapy in a mouse model of pancreatic cancer. Science, 2009. 324(5933): p. 1457-61.

26. Feig, C., et al., Targeting CXCL12 from FAP-expressing carcinoma-associated fibroblasts synergizes with anti-PD-L1 immunotherapy in pancreatic cancer. Proceedings of the National Academy of Sciences of the United States of America, 2013. 110(50): p. 20212-7. 
27. Ijichi, H., et al., Inhibiting Cxcr2 disrupts tumor-stromal interactions and improves survival in a mouse model of pancreatic ductal adenocarcinoma. The Journal of clinical investigation, 2011. 121(10): p. 4106-17.

28. Neesse, A., et al., CTGF antagonism with mAb FG-3019 enhances chemotherapy response without increasing drug delivery in murine ductal pancreas cancer. Proceedings of the National Academy of Sciences of the United States of America, 2013. 110(30): p. 12325-30.

29. Ozdemir, B.C., et al., Depletion of carcinoma-associated fibroblasts and fibrosis induces immunosuppression and accelerates pancreas cancer with reduced survival. Cancer cell, 2014. 25(6): p. 719-34.

30. Rhim, A.D., et al., Stromal elements act to restrain, rather than support, pancreatic ductal adenocarcinoma. Cancer cell, 2014. 25(6): p. 735-47.

31. Frese, K.K., et al., nab-Paclitaxel potentiates gemcitabine activity by reducing cytidine deaminase levels in a mouse model of pancreatic cancer. Cancer discovery, 2012. 2(3): p. 260-9.

32. Uhl, E.W. and N.J. Warner, Mouse Models as Predictors of Human Responses: Evolutionary Medicine. Current pathobiology reports, 2015. 3(3): p. 219-223.

33. Mestas, J. and C.C. Hughes, Of mice and not men: differences between mouse and human immunology. Journal of immunology, 2004. 172(5): p. 2731-8.

34. Calado, R.T. and B. Dumitriu, Telomere dynamics in mice and humans. Seminars in hematology, 2013. 50(2): p. 165-74.

35. Tomasetti, C. and B. Vogelstein, Cancer etiology. Variation in cancer risk among tissues can be explained by the number of stem cell divisions. Science, 2015. 347(6217): p. 7881 .

36. Biankin, A.V., et al., Pancreatic cancer genomes reveal aberrations in axon guidance pathway genes. Nature, 2012. 491(7424): p. 399-405.

37. Fogh, J., J.M. Fogh, and T. Orfeo, One hundred and twenty-seven cultured human tumor cell lines producing tumors in nude mice. Journal of the National Cancer Institute, 1977. 59(1): p. 221-6.

38. Kondo, J., et al., Retaining cell-cell contact enables preparation and culture of spheroids composed of pure primary cancer cells from colorectal cancer. Proceedings of the National Academy of Sciences of the United States of America, 2011. 108(15): p. 623540 .

39. Johnson, J.I., et al., Relationships between drug activity in NCI preclinical in vitro and in vivo models and early clinical trials. British journal of cancer, 2001. 84(10): p. 1424-31. 
40. Voskoglou-Nomikos, T., J.L. Pater, and L. Seymour, Clinical predictive value of the in vitro cell line, human xenograft, and mouse allograft preclinical cancer models. Clinical cancer research : an official journal of the American Association for Cancer Research, 2003. 9(11): p. 4227-39.

41. Hwang, C.I., et al., Preclinical models of pancreatic ductal adenocarcinoma. The Journal of pathology, 2016. 238(2): p. 197-204.

42. Hidalgo, M., et al., Patient-derived xenograft models: an emerging platform for translational cancer research. Cancer discovery, 2014. 4(9): p. 998-1013.

43. Delitto, D., et al., Patient-derived xenograft models for pancreatic adenocarcinoma demonstrate retention of tumor morphology through incorporation of murine stromal elements. The American journal of pathology, 2015. 185(5): p. 1297-303.

44. Gao, H., et al., High-throughput screening using patient-derived tumor xenografts to predict clinical trial drug response. Nature medicine, 2015. 21(11): p. 1318-25.

45. Rubio-Viqueira, B., et al., An in vivo platform for translational drug development in pancreatic cancer. Clinical cancer research : an official journal of the American Association for Cancer Research, 2006. 12(15): p. 4652-61.

46. Fu, X., F. Guadagni, and R.M. Hoffman, A metastatic nude-mouse model of human pancreatic cancer constructed orthotopically with histologically intact patient specimens. Proceedings of the National Academy of Sciences of the United States of America, 1992. 89(12): p. 5645-9.

47. Loukopoulos, P., et al., Orthotopic transplantation models of pancreatic adenocarcinoma derived from cell lines and primary tumors and displaying varying metastatic activity. Pancreas, 2004. 29(3): p. 193-203.

48. Garrido-Laguna, I., et al., Tumor engraftment in nude mice and enrichment in stromarelated gene pathways predict poor survival and resistance to gemcitabine in patients with pancreatic cancer. Clinical cancer research : an official journal of the American Association for Cancer Research, 2011. 17(17): p. 5793-800.

49. Hidalgo, M., et al., A pilot clinical study of treatment guided by personalized tumorgrafts in patients with advanced cancer. Molecular cancer therapeutics, 2011. 10(8): p. 1311-6.

50. Aparicio, S., M. Hidalgo, and A.L. Kung, Examining the utility of patient-derived xenograft mouse models. Nature reviews. Cancer, 2015. 15(5): p. 311-6.

51. Carrel, A. and C.A. Lindberg, The Culture of Organs1938, New York: Paul B. Hoeber, Inc.

52. Jones, R.T., E.A. Hudson, and J.H. Resau, A review of in vitro and in vivo culture techniques for the study of pancreatic carcinogenesis. Cancer, 1981. 47(6 Suppl): p. 1490-6. 
53. Githens, S., 3rd, et al., Ducts of the rat pancreas in a agarose matrix culture. In vitro, 1980. 16(9): p. 797-808.

54. Montesano, R., et al., Collagen matrix promotes reorganization of pancreatic endocrine cell monolayers into islet-like organoids. The Journal of cell biology, 1983. 97(3): p. 935-9.

55. Kerr-Conte, J., et al., Ductal cyst formation in collagen-embedded adult human islet preparations. A means to the reproduction of nesidioblastosis in vitro. Diabetes, 1996. 45(8): p. 1108-14.

56. Marciniak, A., et al., Mouse pancreas tissue slice culture facilitates long-term studies of exocrine and endocrine cell physiology in situ. PloS one, 2013. 8(11): p. e78706.

57. van Geer, M.A., et al., Ex-vivo evaluation of gene therapy vectors in human pancreatic (cancer) tissue slices. World journal of gastroenterology, 2009. 15(11): p. 1359-66.

58. Vaira, V., et al., Preclinical model of organotypic culture for pharmacodynamic profiling of human tumors. Proceedings of the National Academy of Sciences of the United States of America, 2010. 107(18): p. 8352-6.

59. Alagesan, B., et al., Combined MEK and PI3K inhibition in a mouse model of pancreatic cancer. Clinical cancer research : an official journal of the American Association for Cancer Research, 2015. 21(2): p. 396-404.

60. Schreiber, F.S., et al., Successful growth and characterization of mouse pancreatic ductal cells: functional properties of the Ki-RAS(G12V) oncogene. Gastroenterology, 2004. 127(1): p. 250-60.

61. Agbunag, C., et al., Pancreatic duct epithelial cell isolation and cultivation in twodimensional and three-dimensional culture systems. Methods in enzymology, 2006. 407: p. 703-10.

62. Lee, K.E. and D. Bar-Sagi, Oncogenic KRas suppresses inflammation-associated senescence of pancreatic ductal cells. Cancer cell, 2010. 18(5): p. 448-58.

63. Botta, G.P., et al., Constitutive K-RasG12D activation of ERK2 specifically regulates 3D invasion of human pancreatic cancer cells via MMP-1. Molecular cancer research : MCR, 2012. 10(2): p. 183-96.

64. Sempere, L.F., J.R. Gunn, and M. Korc, A novel 3-dimensional culture system uncovers growth stimulatory actions by TGFbeta in pancreatic cancer cells. Cancer biology \& therapy, 2011. 12(3): p. 198-207.

65. Fanjul, M. and E. Hollande, Morphogenesis of "duct-like" structures in threedimensional cultures of human cancerous pancreatic duct cells (Capan-1). In vitro cellular \& developmental biology. Animal, 1993. 29A(7): p. 574-84. 
66. Matsuda, Y., et al., Morphological and cytoskeletal changes of pancreatic cancer cells in three-dimensional spheroidal culture. Medical molecular morphology, 2010. 43(4): p. 211-7.

67. Kim, M.P., et al., ALDH activity selectively defines an enhanced tumor-initiating cell population relative to $C D 133$ expression in human pancreatic adenocarcinoma. PloS one, 2011. 6(6): p. e20636.

68. Wen, Z., et al., A spheroid-based 3-D culture model for pancreatic cancer drug testing, using the acid phosphatase assay. Brazilian journal of medical and biological research = Revista brasileira de pesquisas medicas e biologicas / Sociedade Brasileira de Biofisica ... [et al.], 2013. 46(7): p. 634-42.

69. Yeon, S.E., et al., Application of concave microwells to pancreatic tumor spheroids enabling anticancer drug evaluation in a clinically relevant drug resistance model. PloS one, 2013. 8(9): p. e73345.

70. McLeod, E.J., et al., Multicellular tumor spheroids grown from pancreatic carcinoma cell lines: use as an orthotopic xenograft in athymic nude mice. Pancreas, 1997. 14(3): p. 237-48.

71. Gou, S., et al., Establishment of clonal colony-forming assay for propagation of pancreatic cancer cells with stem cell properties. Pancreas, 2007. 34(4): p. 429-35.

72. Grzesiak, J.J. and M. Bouvet, Determination of the ligand-binding specificities of the alpha2betal and alphalbetal integrins in a novel 3-dimensional in vitro model of pancreatic cancer. Pancreas, 2007. 34(2): p. 220-8.

73. Gutierrez-Barrera, A.M., et al., Establishment of three-dimensional cultures of human pancreatic duct epithelial cells. Biochemical and biophysical research communications, 2007. 358(3): p. 698-703.

74. Hosoya, H., et al., Engineering fibrotic tissue in pancreatic cancer: a novel threedimensional model to investigate nanoparticle delivery. Biochemical and biophysical research communications, 2012. 419(1): p. 32-7.

75. Longati, P., et al., 3D pancreatic carcinoma spheroids induce a matrix-rich, chemoresistant phenotype offering a better model for drug testing. BMC cancer, 2013. 13: p. 95.

76. Ricci, C., et al., Interfacing polymeric scaffolds with primary pancreatic ductal adenocarcinoma cells to develop 3D cancer models. Biomatter, 2014. 4: p. e955386.

77. Froeling, F.E., et al., Organotypic culture model of pancreatic cancer demonstrates that stromal cells modulate E-cadherin, beta-catenin, and Ezrin expression in tumor cells. The American journal of pathology, 2009. 175(2): p. 636-48. 
78. Kadaba, R., et al., Imbalance of desmoplastic stromal cell numbers drives aggressive cancer processes. The Journal of pathology, 2013. 230(1): p. 107-17.

79. Wang, Y.J., et al., Sphere-forming assays for assessment of benign and malignant pancreatic stem cells. Methods in molecular biology, 2013. 980: p. 281-90.

80. Rovira, M., et al., Isolation and characterization of centroacinar/terminal ductal progenitor cells in adult mouse pancreas. Proceedings of the National Academy of Sciences of the United States of America, 2010. 107(1): p. 75-80.

81. Seaberg, R.M., et al., Clonal identification of multipotent precursors from adult mouse pancreas that generate neural and pancreatic lineages. Nature biotechnology, 2004. 22(9): p. 1115-24.

82. Hermann, P.C., et al., Distinct populations of cancer stem cells determine tumor growth and metastatic activity in human pancreatic cancer. Cell stem cell, 2007. 1(3): p. 313-23.

83. Pastrana, E., V. Silva-Vargas, and F. Doetsch, Eyes wide open: a critical review of sphere-formation as an assay for stem cells. Cell stem cell, 2011. 8(5): p. 486-98.

84. Katano, T., et al., Establishment of a long-term three-dimensional primary culture of mouse glandular stomach epithelial cells within the stem cell niche. Biochemical and biophysical research communications, 2013. 432(4): p. 558-63.

85. Bartfeld, S., et al., In vitro expansion of human gastric epithelial stem cells and their responses to bacterial infection. Gastroenterology, 2015. 148(1): p. 126-136 e6.

86. Barker, N., et al., Lgr5(+ve) stem cells drive self-renewal in the stomach and build longlived gastric units in vitro. Cell stem cell, 2010. 6(1): p. 25-36.

87. Ootani, A., et al., Sustained in vitro intestinal epithelial culture within a Wnt-dependent stem cell niche. Nature medicine, 2009. 15(6): p. 701-6.

88. Sato, T., et al., Single Lgr5 stem cells build crypt-villus structures in vitro without a mesenchymal niche. Nature, 2009. 459(7244): p. 262-5.

89. Sato, T., et al., Long-term expansion of epithelial organoids from human colon, adenoma, adenocarcinoma, and Barrett's epithelium. Gastroenterology, 2011. 141(5): p. 1762-72.

90. Huch, M., et al., Long-term culture of genome-stable bipotent stem cells from adult human liver. Cell, 2015. 160(1-2): p. 299-312.

91. Huch, M., et al., In vitro expansion of single Lgr5+ liver stem cells induced by Wntdriven regeneration. Nature, 2013. 494(7436): p. 247-50.

92. Petersen, O.W., et al., Interaction with basement membrane serves to rapidly distinguish growth and differentiation pattern of normal and malignant human breast epithelial cells. 
Proceedings of the National Academy of Sciences of the United States of America, 1992. 89(19): p. 9064-8.

851 93. Schwarz, J.S., H.R. de Jonge, and J.N. Forrest, Jr., Value of Organoids from Comparative 852 Epithelia Models. The Yale journal of biology and medicine, 2015. 88(4): p. 367-74.

853 94. Greggio, C., et al., Artificial three-dimensional niches deconstruct pancreas development 854 in vitro. Development, 2013. 140(21): p. 4452-62.

95. Huch, M., et al., Unlimited in vitro expansion of adult bi-potent pancreas progenitors through the Lgr5/R-spondin axis. The EMBO journal, 2013. 32(20): p. 2708-21.

96. Huch, M. and B.K. Koo, Modeling mouse and human development using organoid cultures. Development, 2015. 142(18): p. 3113-25.

97. Dorrell, C., et al., The organoid-initiating cells in mouse pancreas and liver are

98. Kumar, K., et al., Differential Regulation of ZEB1 and EMT by MAPK-interacting

101. Clevers, H. and E. Bender, Q\&A: Hans Clevers. Banking on organoids. Nature, 2015.

99. Stoker, M.G., M. Shearer, and C. O'Neill, Growth inhibition of polyoma-transformed cells by contact with static normal fibroblasts. Journal of cell science, 1966. 1(3): p. 297310 .

100. van de Wetering, M., et al., Prospective derivation of a living organoid biobank of colorectal cancer patients. Cell, 2015. 161(4): p. 933-45. 521(7551): p. S15.

102. Dekkers, J.F., et al., A functional CFTR assay using primary cystic fibrosis intestinal organoids. Nature medicine, 2013. 19(7): p. 939-45.

103. Schwank, G., et al., Functional repair of CFTR by CRISPR/Cas9 in intestinal stem cell organoids of cystic fibrosis patients. Cell stem cell, 2013. 13(6): p. 653-8.

104. Matano, M., et al., Modeling colorectal cancer using CRISPR-Cas9-mediated engineering of human intestinal organoids. Nat Med, 2015. 21(3): p. 256-62.

105. Drost, J., et al., Sequential cancer mutations in cultured human intestinal stem cells. Nature, 2015. 521(7550): p. 43-7.

106. Bailey, P., et al., Genomic analyses identify molecular subtypes of pancreatic cancer. Nature, 2016. 
107. Weeber, F., et al., Preserved genetic diversity in organoids cultured from biopsies of human colorectal cancer metastases. Proceedings of the National Academy of Sciences of the United States of America, 2015. 112(43): p. 13308-11.

108. Yachida, S., et al., Distant metastasis occurs late during the genetic evolution of pancreatic cancer. Nature, 2010. 467(7319): p. 1114-7.

109. Skardal, A., et al., Liver-Tumor Hybrid Organoids for Modeling Tumor Growth and Drug Response In Vitro. Ann Biomed Eng, 2015. 43(10): p. 2361-73.

110. Eglen, R.M. and D.H. Randle, Drug Discovery Goes Three-Dimensional: Goodbye to Flat High-Throughput Screening? Assay Drug Dev Technol, 2015. 13(5): p. 262-5.

111. Blow, N., Cell culture: building a better matrix. Nature methods, 2009. 6(8): p. 619-622.

112. Gurski, L.A., et al., 3D matrices for anti-cancer drug testing and development. Oncology issues, 2010. January/February: p. 20-25.

113. Gelse, K., E. Poschl, and T. Aigner, Collagens--structure, function, and biosynthesis. Advanced drug delivery reviews, 2003. 55(12): p. 1531-46.

114. Amsterdam, A. and J.D. Jamieson, Studies on dispersed pancreatic exocrine cells. I. Dissociation technique and morphologic characteristics of separated cells. The Journal of cell biology, 1974. 63(3): p. 1037-56.

115. Cirulli, V., et al., Expression and function of alpha(v)beta(3) and alpha(v)beta(5) integrins in the developing pancreas: roles in the adhesion and migration of putative endocrine progenitor cells. The Journal of cell biology, 2000. 150(6): p. 1445-60.

116. Shields, M.A., et al., Biochemical role of the collagen-rich tumour microenvironment in pancreatic cancer progression. The Biochemical journal, 2012. 441(2): p. 541-52.

117. Ohlund, D., et al., Type IV collagen is a tumour stroma-derived biomarker for pancreas cancer. British journal of cancer, 2009. 101(1): p. 91-7.

118. Kleinman, H.K. and G.R. Martin, Matrigel: basement membrane matrix with biological activity. Seminars in cancer biology, 2005. 15(5): p. 378-86.

119. Vukicevic, S., et al., Identification of multiple active growth factors in basement membrane Matrigel suggests caution in interpretation of cellular activity related to extracellular matrix components. Experimental cell research, 1992. 202(1): p. 1-8.

120. Taub, M., et al., Epidermal growth factor or transforming growth factor alpha is required for kidney tubulogenesis in matrigel cultures in serum-free medium. Proceedings of the National Academy of Sciences of the United States of America, 1990. 87(10): p. 4002-6. 
Clovers and Tuveson Labs Pancreatic Ductal Organoids

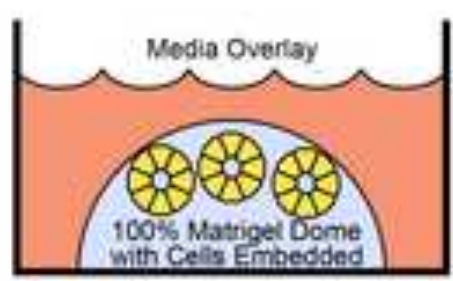

Murine: normal pancreas, PaniN orPDA

Starting

uman normal pancreas, PDA from tumer resection, or FNA

\begin{tabular}{|c|c|}
\hline $\begin{array}{l}\text { Culture } \\
\text { Media }\end{array}$ & 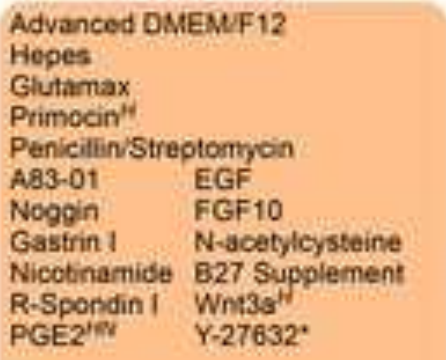 \\
\hline & $\begin{array}{l}\text { MOnly in human media } \\
\text { "Only in human normal media } \\
\text { "Onty when estabilishing thasing }\end{array}$ \\
\hline Matrix & Matrigel \\
\hline Transplant & $\begin{array}{l}\text { Orthotopic } \\
\text { Murine or human tumor organoids } \\
\text { generated neoplasms resembling } \\
\text { PaniNs which progressed to } \\
\text { adenocarcinomas resembling } \\
\text { peimary tumors. Prominent stroma } \\
\text { was present in in transplants. }\end{array}$ \\
\hline $\begin{array}{l}\text { Generation } \\
\text { Efficiency }\end{array}$ & $\begin{array}{l}\text { Muring: }-100 \% \\
\text { Human normal } 75-80 \% \\
\text { Human tumor. } 75-83 \%\end{array}$ \\
\hline Passage & $\begin{array}{l}\text { Murine / human tumor; indefinitely } \\
\text { Human normal: up to } 20 \text { passages }\end{array}$ \\
\hline Freeze & Yes \\
\hline $\begin{array}{l}\text { Targeted } \\
\text { Therapy } \\
\text { Treatment }\end{array}$ & $\begin{array}{l}\text { MNK inhibitor CGP57380 tested: } \\
\text { on human PDA organcids }\end{array}$ \\
\hline
\end{tabular}

Skala Lab

PDA Organoids

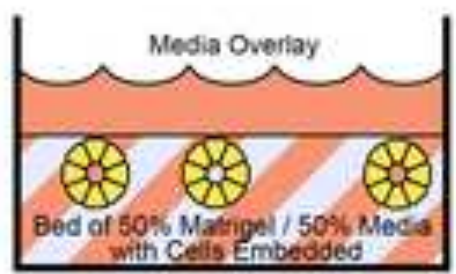

Murine: PDA

Human: PDA from tumor resection

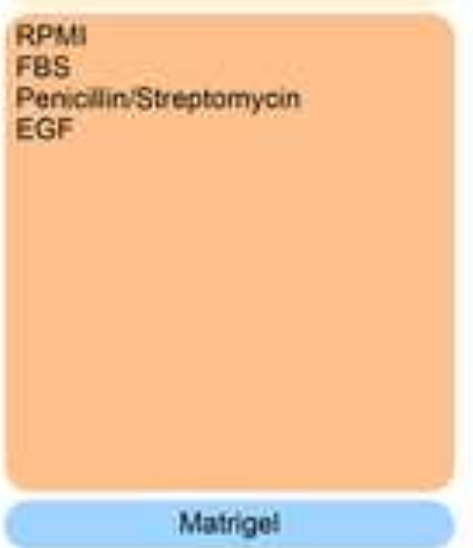

Not Reported

Not Reported

Not Reported

Not Reported

Gemcitatine, JAK2 inhibsor

AZD 1480, MEK inhibitor AZD6244 and PI3K inhibitor XL.147 tested on PDA organoids

Readouts: ocpanoid motabolic and redox state by optical imaging

Muthuswamy Lab Progenitor and PDA Organoids Air-liquid Intorface Organoids

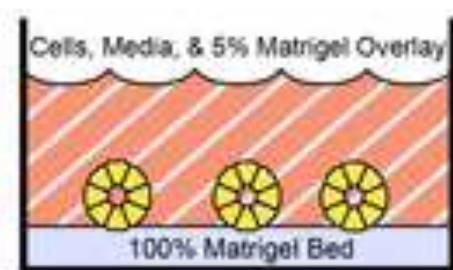

Human pluripotent stem cell.

derived pancreatic progenitors Human POA from tumor resection

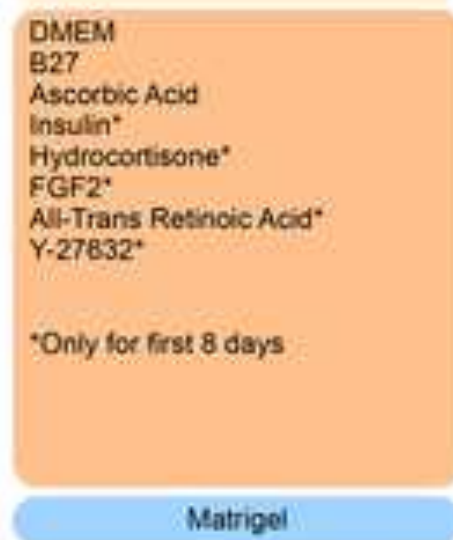

Heterotopic (Mammary Fat Pad) Progeniac organoids expressing KRAS $12 \%$ or TPSSPTYS formed abnormal ductal structures.

Heterotopic (Subcutaneous) Human tumor crganoids generated adenocarcinomas resembing primary tumors.

\section{Progenher. Not Reported} Human tumor: $85 \%$

Progenisor: Not Reported

Human tumor: Yes

\section{Yes}

Gemcitabine and epigentic

therapies tested on human PDA organoids

Readouts; organoid proliferation by MTT assay

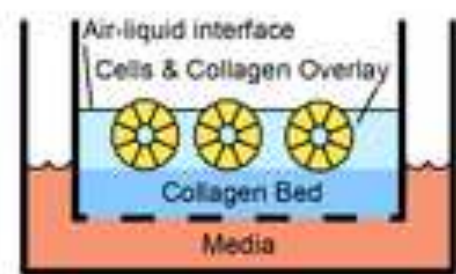

Murine nponatal tissue

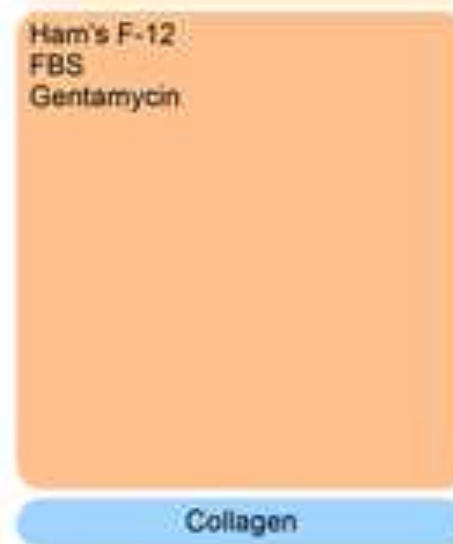

Heterosopic (Subcutaneous) Drganoids expressing Krasorzo andlor lacking $p 53$ generated adenocarcinomas

Not Reported

Onty after activation of Kras6120 or p53 loss

Not Reported

Not Reported 